\title{
Medication-related osteonecrosis of the jaws - a retrospective study
}

\author{
Rossen Kolarov ${ }^{1}$, Daniel Rachev ${ }^{2}$, Desislav \\ Dobrev $^{2}$, Borislava Toncheva ${ }^{2}$
}

1. Department of Oral Surgery, Faculty of Dental medicine, Medical University - Varna;

2. Faculty of Dental medicine, Medical University Varna;

\begin{abstract}
Introduction: Bisphosphonates are used efficiently to treat osteoporosis, bone metastasis of malignant tumors, Paget's disease and multiple myeloma. Bisphosphonate-related osteonecrosis of the jaws (BRONJ) represent a severe side effect, consisting of progressive bone destruction in the maxillofacial region. The aim of this study is to represent clinical information from patients diagnosed with medication-related osteonecrosis of the jaws.

Materials and Methods: A retrospective study was performed over 32 patients hospitalized in the Specialized Hospital for Maxillofacial surgery in Sofia, Bulgaria. All of them diagnosed with osteonecrosis of the jaws. The selection criteria of the patients were based on the existence of previous history for bisphosphonate treatment.

Results: Thirty-one patients were on a bisphosphonate therapy for bone metastasis. Among cancer patients was announced the use of denosumab and zoledronic acid. One patient has received oral treatment with ibandronic acid for osteoporosis. In 23 patients, bone lesions involved the lower jaw, while in 9 patients the lesions persisted in the maxillary bone. Twenty-eight patients were diagnosed with second stage of BRONJ. Exposed bone, purulent secretion, fistulas and swelling were clinically observed among patients. All of the patients received antibiotic treatment for BRONJ, while 2 of them were treated surgically. All patients were signed out after an improvement, as a result from the treatment. Six patients were hospitalized again after time of remission.
\end{abstract}


Conclusion: In conclusion, the study shows that BRONJ is mainly associated with the use of denosumab and zoledronic acid. Most of the patients seek medical attention in the second stage of osteonecrosis. The recommended conservative treatment with antibiotics can lead only to remission in some cases.

\section{Introduction}

Bisphosphonate-related osteonecrosis of the jaws (BONJ) has been recently described as a severe side effect among patients who have a previous history of chronic bisphosphonate treatment. The very first paper describing Bisphosphonate-related osteonecrosis of the jaws (BRONJ) was published in 2003 (1). In 2014 American Association of Oral and Maxillofacial Surgeons (AAOMS) suggested that the nomenclature of BRONJ should be changed to Medication-related osteonecrosis of the jaws (MRONJ), due to the trend of increasing number of cases induced by other antiresorptive and antiangiogenic medicaments (2).

\section{Pathogenesis}

Bisphosphonates are widely used in the management of osteoporosis, malignant bone metastasis, Paget`s disease and multiple myeloma $(3,4)$.

Their proposed mechanisms of action include:

- Inhibition of osteoclast activation and differentiation of their precursors.

- Inhibition of the activity of osteoclasts due to osteoblast-mediated signals.

- Suppressive effect on the function of mature osteoclasts.

- Direct and indirect apoptosis of osteoclasts.

- Inhibition of the dissemination of tumor cells and their adhesion to the bone structures (5).

On the other hand, osteoclasts play an essential role into the process of bone remodeling and bone metabolism. Proposed hypothesis suggest that the predilection of this condition for the jaws is owed to the constant micro trauma during mastication and the persistence of resident bacteria in the oral cavity. Further aberration in bone metabolism and inhibition of the angiogenesis during treatment with bisphosphonates or other antiresorptive medicaments, is consequent to increased chance of developing ONJ (6).

Local factors responsible for the predilection are any periodontal or surgical manipulations onto the dentoalveolar crest (tooth extraction, implants and etc.). Anatomical features, such as palatal torus or thin mucosa make the jaws more susceptible to trauma. (2) In 2005 Aaron P. Sarathy and coworkers, published a paper presenting two cases of MRONJ triggered by endodontic treatment (7).

\section{Incidence}

According to a 2008 study from Aliya Khan, 95\% of the patients with BRONJ have history of high dose intravenous bisphosphonates treatment for bone metastasis, while patients treated with small doses bisphosphonates per os represented only $5 \%$ from the cases (8).

The incidence of medication-related osteonecrosis among patients with oncological condition depends on the dosage and the duration of the treatment (9). Kahn and coworkers concluded in their study that BRONJ occurs in $1-12 \%$ of the cases after 36 months of treatment with bisphosphonates. Furthermore, the incidence of BRONJ among patients, who has gone through oral treatment for several years (etc. treatment of osteoporosis) is less than 1 in 100000 cases per year (8). 


\section{Diagnose and staging}

According to the American Association of Oral and Maxillofacial Surgeons patients can be diagnosed with MRONJ, if the following conditions exist:

- History of previous or current treatment with antiresorbtive or antiangiogenic medicaments.

- No history of radiation therapy in the maxillofacial region.

Exposed bone or bone that can be probed through extra or intraoral fistula in the maxillofacial region that has persisted more than 8 weeks (2).

Ruggiero suggested in 2009 the following classification for staging of MRONJ (10). (Table 1):

\begin{tabular}{c|c}
\hline \hline Table 1. & Suggested by Ruggiero et al at AAOMS in 2009, Clinical Staging of BROJ \\
\hline \hline Stage 0 & $\begin{array}{r}\text { No clinical evidence of necrotic bone but nonspecific } \\
\text { clinical findings and symptoms }\end{array}$ \\
\hline Stage 1 & Exposed/Necrotic bone in patients who are asymptomatic and have no evidence of infection. \\
\hline Stage 2 & $\begin{array}{r}\text { Exposed/Necrotic bone associated with infection as evidenced by pain and erythema in the region of the exposed } \\
\text { bone with or without purulent drainage. }\end{array}$ \\
\hline Stage 3 & $\begin{array}{r}\text { Exposed/Necrotic bone in patients with pain, infection and one or more of the following: Pathologic fracture, extraoral } \\
\text { fistula or osteolysis extending to the inferior border. }\end{array}$ \\
\hline \hline
\end{tabular}

\section{Clinical characteristics}

Exposed bone is common clinical feature of MRONJ. In some complicated cases mobility of the teeth, fistulas, pus discharge and bone fractures can be observed. Clinically, MRONJ can be asymptomatic or can have the characteristics of osteomyelitis. Radiographic characteristics are similar to those in patients with osteomyelitis who have not gone through treatment with antiresorptive medicaments (8).

The study aims to represent a retrospective analysis of group of patients with MRONJ in order to raise awareness among specialists and General Dental Practitioners.

\section{MATERIALS AND METHODS}

For the aims of the study a retrospective chart review was performed on 32 patients referred to the Specialized Hospital for Maxillofacial surgery in Sofia, Bulgaria in the period between 2012 and 2016. All the patients were diagnosed with osteonecrosis of the jaw. Patients were elected to meet the diagnostic criteria proposed by The American Association of Oral and Maxillofacial Surgeons.

\section{RESULTS}

\section{Demographics}

Twenty men $(62,5 \%)$ and twelve women $(37,5 \%)$ took part in the statistical analysis. The average age of the included patients was approximately 64 years (range from 45 to 86 ). 


\section{Treatment of the primary disease}

The most common oncological diagnoses at presenting were prostate and breast cancer. Thirty one of the patients $(96,975 \%)$ with MRONJ announced that they have been taking antiresorptive medicaments as a treatment therapy for bone metastasis of prostate, testicles, colon and breast cancer. Only one patient $(3,125 \%)$ was suffering from osteonecrosis of the jaws due to treatment of osteoporosis with oral bisphosphonates. Five patients declared that they have gone through radiation therapy in a different region from the maxillofacial on the occasion of a primary oncological treatment. According to the medical records, six patients were treated with denosumab (Xgeva), twelve - with Zoledronic acid (Zometa) and one - with both medicaments. One patient has threated osteoporosis with Ibandronic acid (Bonviva) (Graphic 1). The average duration of treatment with antiresorbtive medicaments in patients who have developed MRONJ is 35 months. The results are represented in table 1.

\section{Graphic 1. Treatment of primary disease}

\section{Bonviva (ibandronate) Xgeva (Denosumab) Zometa (Zoledronate) $\square$ Xgeva and Zometa} Bisphosphonate

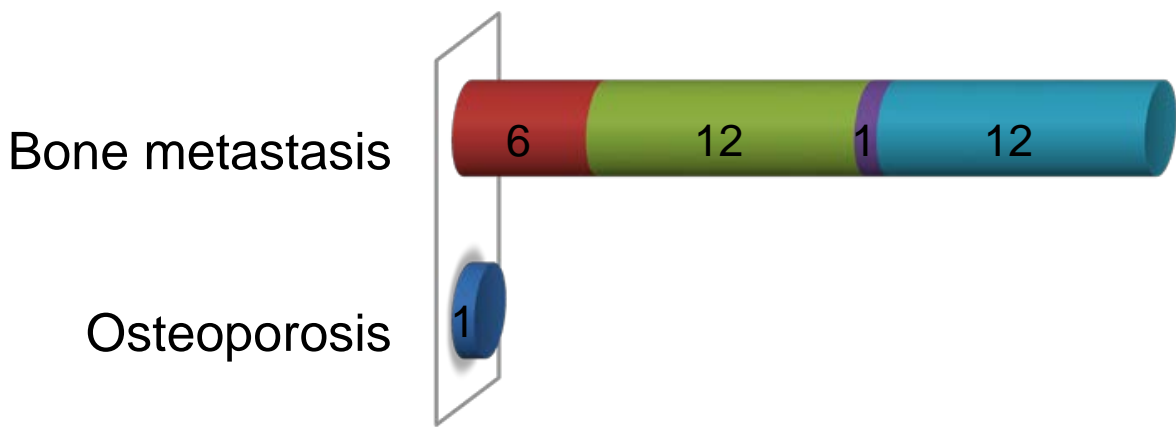

TABLE 1.

\begin{tabular}{|c|c|c|c|c|c|c|c|}
\hline № & Gender & Age & Primary disease & Comorbidity & Time & Medicament & RT \\
\hline 1 & M & 67 & Colon $\mathrm{Ca}$ & $\mathrm{D}, \mathrm{AH}$ & 2 & Bisphoshpnates & - \\
\hline 2 & $\mathrm{~F}$ & 48 & Breast Ca & - & 6 & Denosumab & - \\
\hline 3 & $M$ & 72 & prostate $\mathrm{Ca}$ & $\mathrm{D}, \mathrm{AH}$ & 2 & Bisphoshpnates & - \\
\hline 4 & $\mathrm{~F}$ & 47 & Breast Ca & - & 3 & Zometa & - \\
\hline 5 & M & 64 & prostate $\mathrm{Ca}$ & - & 2 & Zometa & - \\
\hline 6 & $\mathrm{M}$ & 65 & prostate $\mathrm{Ca}$ & $\mathrm{AH}$, Anemia & 2 & Xgeva & - \\
\hline 7 & $\mathrm{~F}$ & 58 & Breast Ca & Allergies & - & Bisphoshpnates & - \\
\hline 8 & $M$ & 79 & prostate $\mathrm{Ca}$ & $\mathrm{D}, \mathrm{AH}$ & 5 & Bisphoshpnates & - \\
\hline
\end{tabular}




\begin{tabular}{|c|c|c|c|c|c|c|c|}
\hline 9 & $\mathrm{~F}$ & 80 & Breast Ca & Asthma & 6 & Zometa & - \\
\hline 10 & $\mathrm{~F}$ & 50 & Breast Ca & Asthma, $\mathrm{AH}$ & 2,5 & Xgeva & - \\
\hline 11 & $\mathrm{~F}$ & 58 & $\mathrm{Ca}$ & - & 3 & Xgeva & - \\
\hline 12 & $\mathrm{~F}$ & 51 & Breast Ca & - & 2 & Zometa & - \\
\hline 13 & M & 83 & prostate $\mathrm{Ca}$ & D, Glaucoma,IHD & 4 & Zometa & + \\
\hline 14 & M & 70 & prostate $\mathrm{Ca}$ & $\mathrm{IHD}, \mathrm{AH}$ & 2 & Zometa & - \\
\hline 15 & M & 54 & prostate $\mathrm{Ca}$ & $\mathrm{AH}$ & 2 & Zometa & + \\
\hline 16 & M & 69 & prostate $\mathrm{Ca}$ & D, Stenocardia & 3 & Bisphoshpnates & - \\
\hline 17 & $\mathrm{~F}$ & 45 & Breast $\mathrm{Ca}$ & - & 5 & Zometa & + \\
\hline 18 & M & 72 & prostate $\mathrm{Ca}$ & - & - & Bisphoshpnates & - \\
\hline 19 & M & 70 & prostate $\mathrm{Ca}$ & $\mathrm{AH}$ & 1 & Bisphoshpnates & - \\
\hline 20 & $\mathrm{~F}$ & 54 & Breast $\mathrm{Ca}$ & $\mathrm{D}$ & 6 & Zometa & - \\
\hline 21 & M & 68 & prostate $\mathrm{Ca}$ & - & 3 & Bisphoshpnates & - \\
\hline 22 & M & 62 & prostate $\mathrm{Ca}$ & - & 5 & Zometa & - \\
\hline 23 & M & 79 & prostate $\mathrm{Ca}$ & $\mathrm{AH}$ & 0,75 & Bisphoshpnates & - \\
\hline 24 & $\mathrm{~F}$ & 61 & Breast Ca & IHD, Podagra, $\mathrm{AH}$ & 4 & Xgeva & - \\
\hline 25 & M & 72 & prostate $\mathrm{Ca}$ & $\mathrm{D}, \mathrm{AH}$ & 2 & Bisphoshpnates & + \\
\hline 26 & M & 86 & prostate $\mathrm{Ca}$ & Stenocardia, $\mathrm{AH}$ & 1 & Xgeva & - \\
\hline 27 & M & 73 & prostate $\mathrm{Ca}$ & $\mathrm{AH}$ & 0,67 & Bisphoshpnates & + \\
\hline 28 & $\mathrm{~F}$ & 54 & Breast Ca & - & 4 & Zometa, Xgiva & - \\
\hline 29 & M & 63 & prostate $\mathrm{Ca}$ & Podagra, Arrhytmia & 2 & Zometa & - \\
\hline 30 & $\mathrm{~F}$ & 46 & Breast Ca & - & - & Zometa & - \\
\hline 31 & M & 55 & Testicle Ca & COPD, Allergies & 1 & Bisphoshpnates & - \\
\hline 32 & M & 71 & Osteoporosis & $\mathrm{D}$ & 2 & Bonviva & - \\
\hline
\end{tabular}

RT - Radiology treatment; M - male; F - female; Ca - cancer; D - diabetes; AH - arterial hypertension; IHD - ischemic heart disease; COPD - chronic obstructive pulmonary disease

\section{Symptoms and physical findings}

Twenty three $(71,875 \%)$ patients presented with mandibular bone involvement, while in 9 patients $(28,125 \%)$ the complication involved the maxillary bone. In regards to the physical manifestations, purulent discharge was observed in 7 patients $(21,875 \%)$, from which one was with extraoral fistula. Facial asymmetry was featured symptom in 9 patients $(28,125 \%)$ and bone sequestration - in 2 patients $(6,25 \%)$. Twenty-eight patients $(87,5 \%)$ presented with complaining of pain in the evolved region and four patients presented with asymptomatic clinical or radiographic findings. According to the early mentioned clinical staging classification, 28 patients were classified as stage 2 of MRONJ and 4 patients as stage 1 (asymptomatic).

\section{Comorbidity and triggering factors}

This group had a large variety of significant comorbidities from which arterial hypertension has found to be the most common (13 patients/ 40,625\%). According to the medical records, $25 \%$ of the patients were affected by diabetes. Nine patients $(28,125 \%)$ presented symptoms of pain and exposed bone at the site 
of previous tooth extraction. Only one patient reported that the symptoms were initiated after placement of implant.

\section{Treatment of MRONJ}

All patients were conservatively treated with antibiotics. In addition to the antibiotic regiment in two of the cases $(6,25 \%)$ was committed sequestrectomy. Five patients $(15,625 \%)$ were treated with Amoxiclav, with Unasyn - $3(9,375 \%)$, with Lyncomicin - $23(71,875 \%)$ and with Amikacin - 1 (3,125\%). Metronidazol was given to 3 patients. Apart from the antibacterial treatment,twenty-four patient were also treated with analgetics (Graphic 2). Improvement in the status was observed in all patients at the moment of their discharge from the hospital (Table 2).

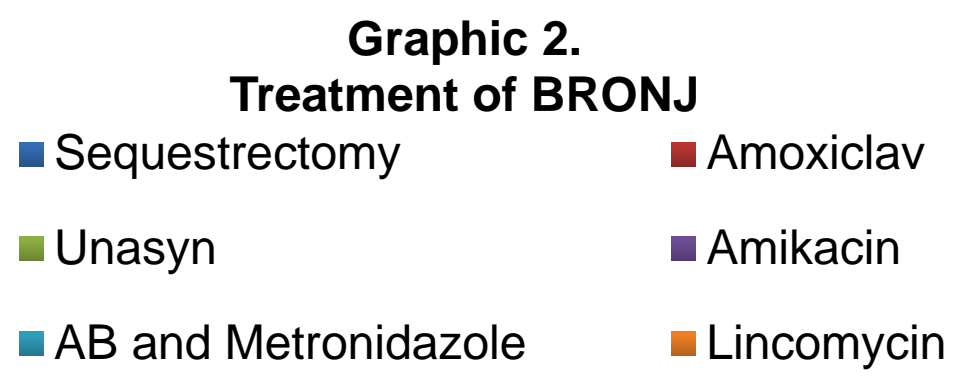

Conservative

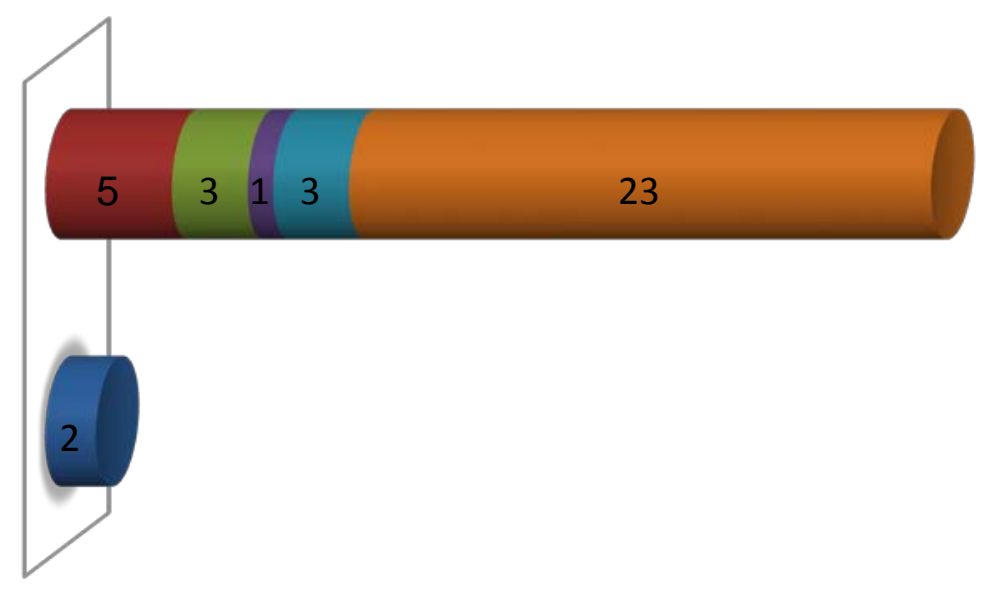

TABLE 2.

\begin{tabular}{|c|c|c|c|c|c|c|c|c|c|}
\hline № & Jaw & $\mathbf{P}$ & $\mathbf{F}$ & $\mathbf{S}$ & F.A. & Stage & S.F. & Treatment & Discharge \\
\hline 1 & Mand. & - & - & - & - & 2 & PEW & Amoxiclav, Analgin, Flagyl & WI \\
\hline 2 & Mand. & - & - & - & - & 2 & PEW, I & Lincomycin, Analgin & WI \\
\hline 3 & Max. & - & - & - & - & 2 & PEW & Amoxiclav, Analgin & WI \\
\hline 4 & Mand. & - & - & - & - & 2 & & Amoxiclav, Analgin & WI \\
\hline 5 & Mand. & - & - & - & - & 1 & & Amoxiclav & WI \\
\hline
\end{tabular}




\begin{tabular}{|c|c|c|c|c|c|c|c|c|c|}
\hline 6 & Mand. & - & - & - & - & 2 & & Unasyn, Analgin & WI \\
\hline 7 & Mand. & - & - & - & + & 1 & & Lincomycin, Analgin & WI \\
\hline 8 & Mand. & - & - & - & - & 2 & & Lincomycin, Analgin, Flagyl & WI \\
\hline 9 & Mand. & + & - & - & + & 2 & PEW & Lincomycin, Analgin & WI \\
\hline 10 & Mand. & - & - & - & - & 2 & & Lincomycin, Analgin & WI \\
\hline 11 & Max. & - & - & - & - & 2 & & Amikacin, Flagyl, Analgin & WI \\
\hline 12 & Max. & + & 10 & - & + & 2 & PEW & Lincomycin & WI \\
\hline 13 & Mand. & - & - & - & - & 2 & & Lincomycin, Analgin & WI \\
\hline 14 & Max. & - & - & - & + & 2 & & Unasyn & WI \\
\hline 15 & Max. & - & - & - & - & 2 & & Unasyn, Analgin & WI \\
\hline 16 & Mand. & - & - & - & - & 2 & PEW & Lincomycin, Analgin & WI \\
\hline 17 & Max. & + & - & + & - & 2 & & Lincomycin, Analgin, surgical & WI \\
\hline 18 & Mand. & + & - & - & + & 2 & & Lincomycin, Analgin & WI \\
\hline 19 & Mand. & - & - & - & - & 1 & & Lincomycin, Analgin & WI \\
\hline 20 & Mand. & - & - & - & + & 1 & & Lincomycin, Analgin & WI \\
\hline 21 & Mand. & - & - & - & - & 2 & & Lincomycin, Analgin & WI \\
\hline 22 & Mand. & - & - & - & - & 2 & PEW & Lincomycin, Analgin & WI \\
\hline 23 & Max. & - & - & - & - & 2 & & Amoxiclav, Analgin & WI \\
\hline 24 & Max. & + & - & - & - & 2 & & Lincomycin, Paracetamol & WI \\
\hline 25 & Max. & - & - & - & - & 2 & PEW & Lincomycin, Analgin & WI \\
\hline 26 & Mand. & - & - & - & - & 2 & PEW & Lincomycin, Analgin, Paracetamol & WI \\
\hline 27 & Mand. & - & - & - & - & 2 & & Lincomycin & WI \\
\hline 28 & Mand. & - & - & - & + & 2 & & Lincomycin, Analgin & WI \\
\hline 29 & Mand. & - & - & - & + & 2 & PEW & Lincomycin, рингер & WI \\
\hline 30 & Mand. & - & - & + & - & 2 & PEW & Lincomycin, sequestrectomy & WI \\
\hline 31 & Mand. & + & - & - & - & 2 & & Lincomycin & WI \\
\hline 32 & Mand. & + & - & - & + & 2 & & Lincomycin & WI \\
\hline
\end{tabular}

P - pus; F - fistula; S - sequestration; FA - facial asymmetry; Max. - Maxilla; Mand. - mandible; PEW - post extraction wound; I - implant; WI - with improvement; IO - intraoral

\section{DISCUSSION}

In the limits of the study one patient was presented with history of oral treatment with bisphosphonates for management of osteoporosis. The low bio-availability of the oral bisphosphonates in the bone matrix is a result of their low intestinal absorption (from 1\% to 10\%), which can be additionally reduced if simultaneously taken with Calcium and Ferrum (4 Intravenously taken bisphosphonates have extended storage of the medicament in the bone. Relevant to the oral intake, the intravenous bisphosphonates show significantly higher incidence of developing osteonecrosis of the jaws $(4,11)$.

According to the medical records, eight patients (25\%) were presented with diabetes. The epidemiological data presented in the study of Khamaisi and coworkers, suggested a possible role of diabetes as a risk factor in the development of osteonecrosis of the jaws. In the limits of their study, eighteen patients (58\%), have developed MRONJ on the background of comorbid diabetes (12). 
According to the medical literature, the hypothetic role of diabetes in the development of MRONJ is mainly associated with microangiopathogenic ischemia of the bones (13). The impaired metabolic processes of the bone result in reduced possibility for bone remodeling and apoptosis of the osteoclast and osteoblast $(14,15)$. The abnormality of the bone metabolism which is presented in patients with comorbid diabetes additionally aggravates the effect of antiangiogenic and antiresorbtive medicaments, resulting in higher chance of developing of osteonecrosis of the bones.

Although, all of the patients were signed out with an improvement in their condition, six of them were referred to the hospital again, due to exacerbation. Two of the exacerbated patients have had complications, classified as stage 3 of osteonecrosis with cutaneous fistula. The conventional treatment with antibiotics and analgesics has been proved to provide satisfying results but not in all cases(16). Additional examination and tracking of the patients is regarded a necessity, in order to evaluate the results from the treatment accurately. A study from V. Aftimos and coworkers suggested positives effect of antifungal medicaments in the conventional management of MRONJ.

\section{Conclusion}

- The incidence of BRONJ among patient on intravenous therapy for bone metastasis is significantly higher than those on oral therapy.

- $\quad$ BRONJ affect the mandibular bone 2,5 times more often than the maxillary bone.

- The most common trigger factor for MRONJ was tooth extraction.

- Diabetes can be related to MRONJ as a predisposing factor.

- In the limits of our study most of the patients with MRONJ seek for medical attention in stage 2 of osteonecrosis.

- The recommended conservative treatment with antibiotics can lead to remission, rather than a full recovery.

\section{References}

1. Marx RE. Pamidronate (Aredia) and Zoledronate (Zometa) Induced Avascular necrosis of the jaws: a growing epidemic. J Oral Maxillofac Surg. 2003;61:1115-1117.

2. Ruggiero SL, Dodson TB, Fantasia J, ET AL.American Association of Oral and Maxillofacial Surgeons Position Paper on Medication-Related Osteonecrosis of the Jaw-2014Update. J Oral Maxillofac Surg 2014;72:1938-1956.

3. Drake MT, Clarke BL, Khosla S. Bisphosphonates: Mechanism of Action and Role in Clinical Practice. Mayo Clin Proc 2008;83:1032-1045.

4. Martin T. Experimental and Clinical Pharmacology: Bisphosphonates - Mechanisms of Action. Australian Prescriber 2000;23:6:130-132.

5. Ralte S, Bhattacharyya A. Effect of Bisphosphonate on Osteoclast of Bone. Forensic Medicine and Anatomy Research 2014;2:3:56-62.

6. Rasmusson L, Jahan A. Bisphosphonate Associated Osteonecrosis of the Jaw: An Update on Pathophysiology, Risk Factors, and Treatment.International Journal of Dentistry 2014; 1-9.

7. Sarathy AP, Bourgeois SL Jr, Goodell GG.Bisphosphonate-Associated Osteonecrosis of the Jaws and Endodontic Treatment: Two Case Reports. J Endod 2005;10:759-763.

8. Kahn A, Sandor G, Dore E, et al..Bisphosphonate associated osteonecrosis of the jaw. The Journal of Rheumatology 2009;36:478-490.

9. Bamias A, Kastritis E, Bamia C, et al. Osteonecrosis of the Jaw in Cancer After Treatment With Bisphosphonates: Incidence and Risk Factors. Journal of Clinical Oncology 2005;23:34:8580-8587.

10. Ruggiero SL, Dodson TB, Assael LA, et al. American Association of Oral and Maxillofacial Surgeons. American Association of Oral and Maxillofacial Surgeons position paper on bisphosphonaterelated osteonecrosis of the jaws - 2009 update. J Oral Maxillofac Surg 2009;67:5:2-12. 
11. Baraldi A, Fusco V, Bellora A, et al. Prevention of Bisphosphonates-Induced Osteonecrosis. Clinical Cases in Mineral and Bone Metabolism 2007;4:1:58-61.

12. Khamaisi, Mogher, Eran Regev, Noam Yarom, Batia Avni, Eran Leitersdorf, Itamar Raz, and Sharon Elad. "Possible Association between Diabetes and Bisphosphonate-Related Jaw Osteonecrosis. The Journal of Clinical Endocrinology \& Metabolism 2007;3:1172-1175.

13. Hill SL, Holtzman GI, Buse R. The Effects of Peripheral Vascular Disease with Osteomyelitis in the Diabetic Foot. American Journal of Surgery 1999;177:4:282-286.

14. Carnevale V, Romagnoli E, D'Erasmo E. Skeletal Involvement in Patients with Diabetes Mellitus. Diabetes/Metabolism Research and Reviews 2004;20:3:196-204.

15. Kumeda Y. Bone Metabolic Abnormality in Diabetes: Especially about Osteoblast Dysfunction. Clin Calcium. 2006; 8:1277-1285.

16. Nomura T, Shibahara T, Uchiyama T, et al. Bisphosphonate-Related Osteonecrosis of Jaw (BRONJ) in Japanese Population: A Case Series of 13 Patients at Our Clinic. The Bulletin of Tokyo Dental College 2013;54:117-125.

\section{Corresponding author:}

Rossen Kolarov,

Faculty of Dental Medicine,

Department of Oral and Maxillofacial Surgery and

Specialized Imaging Diagnostics,

Medical University-Varna 